\title{
Multidisciplinary Design of a micro-USV for Re-entry Operations
}

\author{
Edmondo Minisci* Hou Liqiang †, Massimiliano Vasile \\ University of Glasgow, Glasgow, G12 8QQ, United Kingdom
}

\begin{abstract}
This paper presents the robust multi-disciplinary design of a small scale unmanned space vehicle for re-entry operations. Uncertainties on the aerodynamic forces, thermal flux and characteristics of the thermal protection material are incorporated into the vehicle model. A Monte-Carlo sampling procedure is used to compute the mean and variance of the maximum heat flux and internal temperature. Then a population-based multi-objective optimization algorithm of the EDA type (Estimation of Distribution Algorithms) searches for geometries that minimize the mean value of the maximum heat flux, mean value of the maximum internal temperature, and the weighted sum of their variance. The EDA is hybridized with a direct transcription method for optimal control problems. The evolutionary part handles the shape parameters of the vehicle and the uncertain objective functions, while the direct transcription method generates an optimal control profile for the re-entry trajectory. A multi-fidelity approach is adopted during the optimization process to estimate the correct aerodynamic properties of the vehicle. Initially a low-fidelity analytical model, fitted on a wave-rider type of vehicle, is used to train an artificial neural network (ANN) which approximates the aerodynamic forces. Through the evolution a mix of analytical and CFD high-fidelity calculations are used to update the ANN. CFD runs progressively become the main source of updates for the ANN till, close to convergence of the EA, the analytical model is almost completely dropped. Some preliminary results show the achievable performance of such a small spacecraft and the requirements in terms of thermal protection materials.
\end{abstract}

\section{Introduction}

Unmanned Space Vehicles (USV) are seen as a test-bed for enabling technologies and as a carrier to deliver and return experiments to and from low-Earth orbit. USV's are a potentially interesting solution also for the exploration of other planets or as long-range recognisance vehicles. As test bed, USV's are seen as a stepping stone for the development of future generation re-usable launchers but also as way to test key technologies for re-entry operations.

Examples of recent developments are the PRORA-USV,${ }^{1-3}$ designed by the Italian Aerospace Research Center (CIRA) in collaboration with Gavazzi Space, or the Boeing X-37B Orbital Test Vehicle (OTV), that is foreseen as an alternative to the space shuttle to deliver experiments into Earth orbit. Among the technologies to be demonstrated with the X-37 are improved thermal protection systems, avionics, the autonomous guidance system, and an advanced airframe.

Following the path that brought from satellites to current micro cube satellites, this paper presents one first attempt to design a small scale USV. The goal is to derive the technical specifications for a small and relatively inexpensive vehicle which could be used as a technological demonstrator. The design is carried out taking into account the availability of last generation thermal protection systems (TPS) based on ultra-high temperature ceramic materials (UHTC), which are currently considered for the design of the

\footnotetext{
*Research Fellow, Space Advanced Research Team, Department of Aerospace Engineering, James Watt Building South, University of Glasgow, email: e.minisci@aero.gla.ac.uk.

$\dagger$ Visiting Scholar, The State Key Laboratory of Astronautics Dynamics (ADL), Xi'an Satellite Control Center, China, email: houliqiang2008@hotmail.com

${ }^{\ddagger}$ Senior Lecturer, Space Advanced Research Team, Department of Aerospace Engineering, James Watt Building South, University of Glasgow, email: m.vasile@aero.gla.ac.uk, AIAA Senior Member.
} 
PRORA vehicle. ${ }^{4,5}$ UHTC are a family of ceramic materials with extremely high melting temperatures, good oxidation resistance in reentry type environments, and reasonably good thermal shock resistance, which can be used to protect small radius edges of the new generation high efficiency spacecrafts.

This paper presents the robust design optimization of a small scale USV, whose shape is based on an ideal waverider configuration ${ }^{6-8}$ ). The geometry of the ideal waverider is here modified in order to introduce more realistic rounded edges.

Robust design optimization is implemented by means of an incremental single process (ISP) approach, which allows for dealing with the complexity and computational costs of the multidisciplinary optimization process. The ISP consists of a single integrated optimization, in which the initial iterations are performed with a low fidelity model, and the complexity and fidelity of the model are progressively increased during the optimization process. ${ }^{9-12}$

Since the USV will go through different regimes of motion, from hypersonic, in thin atmosphere, to supersonic and subsonic, as it approaches the Earth surface, the fidelity of the model is increased differently depending on the regime. A meta-modeling technique based on Artificial Neural Networks approximators, is used to reduce the cost of aerodynamic computations. During the optimization process, the aerodynamic database used to generate the meta-model is updated with the outcomes of models with different fidelity. ${ }^{13,14}$ In particular, low-fidelity ones will be used to generate samples globally over the range of the design parameters, while high fidelity models will be used to refine the meta-model locally, in later stages of the optimization.

The optimization process is based on an evolutionary algorithm combining global and local search. At every step of the evolutionary algorithm, an optimal control problem, associated to each individual in the population, is solved with a direct transcription method. Two direct transcription methods were tested: finite elements in time defined on spectral basis ${ }^{15,16}$ and Gauss pseudospectral methods. ${ }^{17}$ Both methods are similar in nature and perform similarly on this problem.

The paper starts by describing the aerodynamic, thermal and dynamic models of the vehicle. Then introduces an approach to robust multidisciplinary design based on ISP, together with the modeling and treatment of model uncertainties. Some preliminary results show the achievable performance of this type of vehicle and a trade-off between optimality and robustness.

\section{USV System Models}

This section introduces all system models used to compute the characteristics of the vehicle: geometry, aerodynamic forces, heat flux, dynamics and kinematics.

\section{II.A. Geometry and Shape Model}

The vehicle is a modified version of a waverider with rounded edges. The waverider baseline geometry is defined by three two-dimensional power-law equations. ${ }^{8}$ The planform and the upper surfaces of the vehicle is parameterized by the length $l$, the width, $w$, a power law exponent $n$, the vehicle center line wedge angle, $\theta$, and $\beta$, which is the oblique shockwave inclination angle. ${ }^{8}$ An example can be found in Fig. 1 , where the original wiverider sharp-edge shape is modified to introduce a rounded edge with radius of curvature, $R_{n}>0$. For the example in Fig. 1 the parameters defining the shape are: $l=1.0 m, w=0.8 m, n=0.3, \theta=10 \mathrm{deg}, \beta=$ 12 deg, $R_{n}=0.02 m$.

\section{II.B. Aerodynamic Models}

Two different models are used to predict the aerodynamic characteristics of the vehicle. The former one is a simplified analytical model, which is here applied to the actual rounded-edge vehicle, although it was originally developed to predict the aerodynamics of the original sharp-edge shape of the waverider configuration, in order to have a very first approximation of the performance at the early stage of the design process. The latter one is a full high-fidelity computational fluid dynamic (CFD) model based on a finite volume integration of Reynolds Averaged Navier-Stokes equations (RANS). 


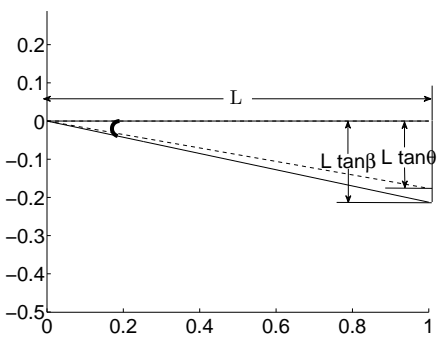

(a) Side View

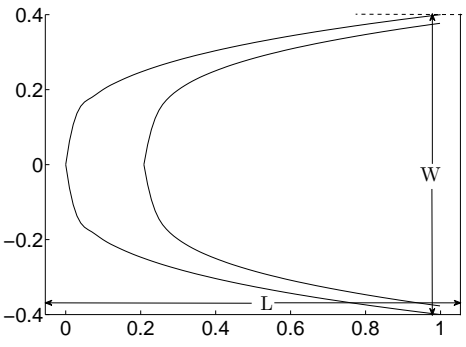

(b) Top View

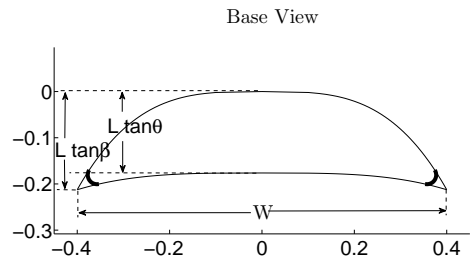

(c) Base View

Figure 1: Example of Vehicle Geometry: $l=1.0 \mathrm{~m}, w=0.8 \mathrm{~m}, n=0.3, \theta=10 \mathrm{deg}, \beta=12 \mathrm{deg}, R_{n}=0.02 \mathrm{~m}$

\section{II.B.1. Analytic Hypersonic Model}

The analytic model gives the lift $L$ and wave $\operatorname{drag} D_{w}$ as functions of the pressure on the upper, lower and base surfaces, $P_{u}, P_{l}$ and $P_{b}$, respectively:

$$
\left\{\begin{array}{l}
L=S_{b}\left(P_{b}-P_{l}\right) \sin \alpha+S_{p}\left(P_{b}-P_{l}\right) \cos \alpha \\
D_{w}=S_{b}\left(P_{l}-P_{b}\right) \cos \alpha+S_{p}\left(P_{l}-P_{u}\right) \sin \alpha
\end{array}\right.
$$

where $S_{p}$ and $S_{b}$ are the planform area and the area of the base, computed as:

$$
\left\{\begin{aligned}
S_{p} & =\frac{w l}{n+1} \\
S_{b} & =S_{p} \tan (\theta)
\end{aligned}\right.
$$

The pressure on the surfaces can be calculated analytically with the oblique shock theory or PrandtlMeyer expansion theory. ${ }^{18}$

The viscous drag $D_{v}$ is given in analytical form, by using the reference temperature method, ${ }^{19}$ as:

$$
\left\{D_{v, \text { surf }}=G_{1} w F(n)\left(\frac{l}{\cos \theta_{\text {surf }}}\right)^{G_{2}}\right.
$$

with $F(n)=F_{0}+F_{1} n+F_{2} n^{2}+F_{3} n^{3}$, and $\theta_{\text {surf }}$ is the inclination angle for the considered surface (upper or lower surface). The adopted constants, which depends on the flow conditions, are given in table 1 .

Table 1: Viscous drag constants for wedge-derived waverider

\begin{tabular}{lcc}
\hline \hline Constants & Laminar flow & Turbulent flow \\
\hline$G_{1}$ & $0.664 \sqrt{\rho_{e}^{2} v_{e}^{3} \mu^{*} C^{*} / \rho^{*}}$ & $0.037 v_{e}^{1.8}\left(\rho^{*}\right)^{0.8}\left(\mu^{*}\right)^{0.2}$ \\
$G_{2}$ & 0.5 & 0.8 \\
$F_{0}$ & 0.99845 & 0.99758 \\
$F_{1}$ & -0.57529 & -0.80941 \\
$F_{2} 0.36737$ & 0.54989 & \\
$F_{3}$ & -0.11939 & -0.18247 \\
\hline \hline
\end{tabular}

In Table 1 , the Chapman-Rubensin parameter $C^{*} \approx\left(T_{*} / T_{e}\right)^{-\frac{1}{3}}, \rho^{*}$, and $\mu^{*}$ are determined by Eckert's empirical relation and Sutherland viscosity law, while $\rho_{e}, T_{e}$ and $v_{e}$ and are the density, the temperature and the flow velocity at edge of boundary layer in post-shock conditions.

The total drag $D$ is $=D_{w}+D_{v, u}+D_{v, l}$, where $D_{v, u}$ and $D_{v, l}$ are the viscous drag of the upper and lower surface, respectively.

As previously anticipated, this simplified model still considers the sharped shape of the wave rider, and does not take into account the modified one, with the introduction of the rounded edges. 


\section{II.B.2. CFD Model}

A commercial code (Numeca $\AA$ ), solving the Reynolds Averaged Navier-Stokes (RANS) equations, was used to obtain an improved solution when the analytical model could be applied and to compute a solution when the analytical model could not be applied (for super- and sub-sonic flight regimes).

With Numeca, the computational domain was discretized by a multi-block structured mesh, changed by internal scripting on the basis of design parameters, made by 13 blocks with near $1.210^{6}$ total nodes. Since no out of plane flight conditions will be considered, the domain discretizes only half of the actual domain and mirror plane conditions are imposed into the longitudinal plane.

Four different settings are implemented and used during the process:

- Laminar Hypersonic for Mach $>6$ and Reynolds number $<10^{5}$

- Fully turbulent (no transition model is considered) Hypersonic for Mach $>6$ and Reynolds number $>10^{5}$

- Laminar Supersonic and Subsonic for Mach $<6$ and Reynolds number $<10^{5}$

- Fully turbulent Supersonic and Subsonic for Mach $<6$ and Reynolds number $>10^{5}$

For hypersonic conditions the radiative equilibrium temperature (see next section) a the nose is imposed on the solid. Real gases database is enhanced on the basis of reported air data for high temperatures. ${ }^{20}$

\section{II.C. TPS and Thermal Model}

The thermal protection system (TPS) is assumed to be made of Zirconium Diboride $\left(Z r B_{2}\right)$ UHTC, which has thermal properties as in Table $2 .^{21}$

Table 2: Zbr2 properties

\begin{tabular}{ccc}
\hline \hline Properties & & unit \\
\hline density & 6000 & $\mathrm{~kg} / \mathrm{m}^{3}$ \\
specific heat & 628 & $\mathrm{~J} \mathrm{Kg}^{-1} \mathrm{~K}^{-1}$ \\
thermal conductivity & 66 & $\mathrm{~W} \mathrm{~m}^{-1} \mathrm{~K}^{-1}$ \\
emissivity & 0.8 & \\
\hline \hline
\end{tabular}

For this preliminary study the highest heat flux is expected to be at the USV nose cap. Thus, the whole nose cone is made of UHTC with thickness $L_{T P S}$. The rest of the vehicle is covered with a thin shell with a constant thickness of $0.002 m$ (similarly to what proposed in Ref. ${ }^{21}$ ).

For the design process, the convective heat flux is computed in the simplest way, with the analytical formula: ${ }^{22}$

$$
\dot{q}_{c o n v}=K_{e} \sqrt{\frac{\rho_{\infty}}{R_{n}}} V_{\infty}^{3}
$$

where $K_{e}=1.742 e-4$, and the heat flux $\dot{q}_{c o n}$ is in $w / m^{2}$.

The internal temperature $\left(T_{\text {int }}\right)$ is computed by solving the following one-dimensional heat equation:

$$
\frac{\partial^{2} T}{\partial x^{2}}=\frac{c_{v} \rho_{T P S}}{k} \frac{\partial T}{\partial t}
$$

with boundary conditions:

$$
\begin{aligned}
& \dot{q}_{c o n v}-\epsilon \sigma T_{w}^{4}+k \frac{d T}{d x} \quad \text { at } \quad x=0 \\
& k \frac{d T}{d x}=\epsilon \sigma T_{\text {int }}^{4} \quad x=L_{T P S}
\end{aligned}
$$

where $c_{v}$ is the heat capacity, $\rho_{T P S}$ is the density of the TPS material, $k$ is the thermal conductivity, $\epsilon$ is the material emissivity, and $\sigma$ is the Stephen-Bolzmann's constant. ${ }^{23}$ Note that no dissipation through 
the structure is considered and no radiation on the back of the vehicle either. The internal temperature is therefore overestimated and depends only on the thickness of the TPS and on the total input heat. Therefore, a re-entry trajectory with a short arc with high $\dot{q}_{c o n v}$ might have an internal temperature which is lower than a trajectory with a long arc with lower heat flux.

\section{II.D. Mass Model}

The total mass of the USV is made of the structural mass $m_{s t}$ the mass of the TPS $m_{T P S}$ and the mass of the payload (avionics and power system) $m_{p l}$.

$$
m=m_{T P S}+m_{s t}+m_{p l}
$$

The mass of the payload is here assumed to be about $20 \%$ of the structural mass, therefore $m_{p l}=0.2 m_{s t}$. The mass of the TPS is made of the mass of the nose $m_{\text {nose }}=\rho_{T P S} V_{n}$ plus the mass of the thin skin covering the rest of the vehicle $m_{\text {skin }}$, where $V_{n}$, is the volume of the nose and $\rho_{T P S}$ the density of the TPS material. The mass of the TPS skin covering the vehicle, except the nose, is:

$$
m_{\text {skin }}=\rho_{\mathrm{tps}} S_{T P S} d_{T P S}
$$

where $d_{T P S}$ is the thickness of the TPS, and $S_{T P S}$ is surface area except that of the nose, which can be approximated by $S_{T P S}=2 S_{p}+S_{b}-S_{n}\left(S_{p}\right.$ is the total planform surface and $S_{b}$ is the area of the rear part, and $S_{n}$ is the surface of the TPS nose cone).

The structure of the vehicle is supposed to be made of titanium, with a density of $4000 \mathrm{~kg} / \mathrm{m}^{3}$. The structural mass $m_{s t}$, can be obtained from:

$$
m_{s t}=\rho_{\mathrm{body}}\left(2 S_{p}+S_{b}\right) d_{b o b y}
$$

where, in this case, $d_{\text {boby }}=0.004 \mathrm{~m}$ is the thickness of the structure of the vehicle, seen as a shell.

\section{II.E. Dynamic Equations and Optimal Control Subproblem}

The vehicle is considered to be a point mass, whose motion is governed by the following set of dynamic equations: ${ }^{24}$

$$
\left\{\begin{array}{l}
\dot{r}=v \sin \theta_{p} \\
\dot{\lambda}=\frac{v \cos \theta_{p} \cos \xi}{r \cos \phi} \\
\dot{\phi}=\frac{v \cos \theta_{p} \sin \xi}{r} \\
\dot{v}=-\frac{D(\alpha)}{m}-g \sin \theta_{p} \\
\dot{\theta_{p}}=\frac{L(\alpha)}{m v} \cos \gamma_{v}-\left(\frac{g}{v}-\frac{v}{r}\right) \cos \theta_{p} \\
\dot{\xi}=\frac{L(\alpha)}{m v \cos \theta} \sin \gamma_{v}-\frac{v}{r} \cos \theta_{p} \cos \xi \tan \phi
\end{array}\right.
$$

where $r$ is the norm of the position vector with respect to the center of the planet, $\lambda$ is the longitude, $\phi$ the latitude, $v$ the magnitude of the velocity, $\theta_{p}$ is the flight path angle, $\xi$ is the heading angle (azimuth of the velocity). No out of plane maneuvers are considered, thus $\gamma_{v}$ is kept equal to zero during the whole trajectory. The angle of attack $\alpha$ is the control variable therefore for each geometry the following optimal control subproblem needs to be solved:

$$
\min _{\alpha} \max _{t} \dot{q}
$$


subject to dynamic equations (10) and terminal conditions:

$$
\left\{\begin{array}{l}
r(t=0)=r_{0} \\
\lambda(t=0)=\lambda_{0} \\
\phi(t=0)=\phi_{0} \\
v(t=0)=v_{0} \\
\theta_{p}(t=0)=\theta_{0} \\
\xi(t=0)=\xi_{0} \\
r\left(t=t_{f}\right) \leq r_{f} \\
r\left(t=t_{f}\right) \geq r_{\min }
\end{array}\right.
$$

The re-entry time is free and no other terminal conditions are imposed as there is no specific requirement on the landing point. Note that thermal equation (5) is solved only once an optimal trajectory is obtained from the solution of problem (11). In fact, every optimal control profile is re-propagated forward in time an heat flux and internal temperature are computed with Eq. (5) and Eq.(4).

\section{Robust Multidisciplinary Design Approach}

The design of the micro-USV requires the simultaneous optimization of the shape and trajectory control profile of the vehicle. In fact, both of them have an impact on the maximum heat flux that the vehicle has to withstand and therefore on its material, mass and size.

The approach taken in this paper hybridizes an evolutionary multi-objective algorithm with a direct transcription method for optimal control problems. The evolutionary part handles the shape parameters and the global optimization of the performance indexes, i.e. heat flux, thermal load and their variance. The performance index of each individual in the population are the results of the optimal control profile coming from the solution of a nonlinear programming problem. The trajectory optimization part of the algorithm relies on an artificial neural network, which approximates the aerodynamic forces acting on the vehicle. The aerodynamic forces are a function of the shape of the vehicle and its operation conditions, such as the angle of attack, $\alpha$, the speed, $v$, and the altitude $H$ (defined $H=r-R_{E}$, where $R_{E}$ is the mean radius of the Earth). In order to reduce the computational costs related to the training and updating of the ANN, a multi-fidelity incremental approach is adopted.

\section{III.A. Robust Design Optimization Under Uncertainty}

The USV is designed to follow a re-entry trajectory that minimizes the heat flux. A second performance index is the internal temperature that together with the heat flux defines the mass of the TPS.

Optimal control problem (11) considers the deterministic value of the maximum heat flux coming from an optimal $\alpha$ profile. However, a number of model parameters can be considered uncertain. In particular, the aerodynamic forces and the characteristics of the thermal protection material.

Therefore, one can associate to the nominal value of lift $L_{d e t}$ and $\operatorname{drag} D_{\text {det }}$, the uncertain quantities;

$$
\begin{aligned}
& L_{u n c}=L_{d e t}+\operatorname{Err}(\alpha, v, H) C_{E}(\alpha, v, H) L_{\text {det }} \\
& D_{u n c}=D_{d e t}+\operatorname{Err}(\alpha, v, H) C_{E}(\alpha, v, H) D_{d e t}
\end{aligned}
$$

where $E r r$ is an error function, which depends on the angle of attack, the speed and the altitude $H$ and $C_{E}$ is a sampling hyper-surface which maps a triplet of values of angles of attack, speed and altitude into the interval $[-1,1]$. Since the idea is that the uncertainties of the aerodynamic data increase with the angle of attack, speed, and altitude, then Err is modeled here as a linear 3D surface, with values that varies from 0.2 , when angle of attack, speed and altitude are $=0$, to 0.8 , when the incidence is $=20 \mathrm{deg}$, the speed is $=8000 \mathrm{~m} / \mathrm{s}$ and the altitude is $100 \mathrm{~km}$. Some of the characteristics of the thermal protection material are considered uncertain as well. In particular the thermal conductivity, $k$, and the specific heat, $c_{p}$ can uniformly vary in the range \pm 0.1 of the reference value.

Thus, given a nominal trajectory with an optimal control profile $\alpha^{*}, N_{s}$ variated values were generated for $L, D, k$ and $c_{p}$ and used to re-propagate the trajectory, maintaining the control profile constant, and 
re-compute heat flux and internal temperature. Then, the mean, $E_{q}$ and $E_{T}$, and the variance, $\sigma_{q}^{2}$ and $\sigma_{T}^{2}$, of all the computed maximum heat flux and maximum internal temperatures were used as performance indexes.

Based on this definition of the performance indexes, the robust design optimization under uncertainties can be formulated as follows:

$$
\min _{\mathbf{d} \in D}\left[E_{q}, E_{T}, \sigma_{q}^{2}, \sigma_{T}^{2}\right]
$$

subject to the following constraints on the variance:

$$
\sigma_{q}^{2} \leq \overline{\sigma_{q}^{2}} ; \sigma_{T}^{2} \leq \overline{\sigma_{T}^{2}}
$$

the design vector $\mathbf{d}$ is defined as follows

$$
\mathbf{d}=\left[l, w, n, \theta, R_{n}, L_{T P S}\right]
$$

the design domain $D$ will be define more precisely in the following section.

Note that a precise model of the uncertainties should also consider other factors, such as: a) the fidelity of the model (analytical vs. CFD), b) the quality of the solution (e.g. relative quality of the mesh for CFD calculations, convergence level of the CFD runs and the NLP problem), and c) meta-modeling approximation error introduced by the ANN. All these aspects are under current investigation and will be presented in future works.

\section{III.B. Multi-objective Algorithm}

MOO problem (14) was solved with a particular type of evolutionary algorithm which belongs to the sub-class of Estimation of Distribution Algorithms (EDAs). EDAs derive from one of the ways researchers thought they could overcome difficulties in finding good solutions for complex problem by simpler evolutionary, genetic algorithms. Generally, these methods, starting from the current population, build a probabilistic model of the search space, then crossover and mutation operators are replaced by a sampling procedure that operates on the probabilistic model. EDAs ensure an effective mixing and reproduction of solution sub-structures due to their ability to accurately capture the structure of a given problem.

The specific EDA employed in this work is derived from the MOPED (Multi-Objective Parzen based Estimation of Distribution) algorithm. ${ }^{25,26}$ MOPED is a multi-objective optimization algorithm for continuous problems that uses the Parzen method to build a probabilistic representation of Pareto optimal solutions, with multivariate dependencies among variables. Non-dominated sorting and crowding operators ${ }^{27}$ are used to classify promising solutions in the objective space, while new individuals are obtained by sampling from the Parzen model.

The Parzen method ${ }^{28}$ is a non-parametric approach to kernel density estimation which gives rise to an estimator that converges everywhere to the true Probability Density Function (PDF) in the mean square sense. Should the true PDF be uniformly continuous, the Parzen estimator can also be made uniformly consistent. In short, the method allocates exactly $n_{k}$ identical kernels, each one centered on a different element of the sample. More details on the original code can be found in the cited works. In the next section the trajectory optimization code, and the evolutionary control technique with multi-fidelity are detailed.

\section{III.B.1. Trajectory Optimization}

Problem (11) with constraint Eqs. (10) and (12) was transcribed with a Gauss pseudospectral method and with Finite Elements in Time on spectral basis. ${ }^{15}$ The two approaches gave similar results therefore it was decided to omit from this paper the comparison between the two approaches on this particular problem. In both cases, the trajectory is decomposed in $N$ elements, each of which have $n_{p}$ collocation points. After transcription, the optimal control problem defined by (11), (10) and (12) becomes the following general nonlinear programming problem:

$$
\min _{\alpha_{s}} \max _{t_{s}} \dot{q}_{s}
$$

subject to the nonlinear algebraic constraints:

$$
C\left(r_{s}, \lambda_{s}, v_{s}, \xi_{s}, \theta_{s}, \alpha_{s}, t_{s}\right)=0
$$


and the terminal constraints;

$$
\left\{\begin{array}{l}
r(t=0)=r_{0} \\
\lambda(t=0)=\lambda_{0} \\
\phi(t=0)=\phi_{0} \\
v(t=0)=v_{0} \\
\theta_{p}(t=0)=\theta_{0} \\
\xi(t=0)=\xi_{0} \\
r\left(t=t_{f}\right) \leq r_{f} \\
r\left(t=t_{f}\right) \geq r_{\min }
\end{array}\right.
$$

where $r_{s}, \lambda_{s}, v_{s}, \xi_{s}, \theta_{s}, \alpha_{s}, t_{s}$ are the discrete values of the time, states and control values at the nodes of the transcription scheme. The NLP problem was solved with the Matlabßfunction fmincon. Two different solution algorithms were tested: active-set and interior-point. The latter demonstrated a superior robustness and convergence speed.

\section{III.C. Evolutionary Control and Multi-fidelity Approach}

The basic idea of the evolutionary control approach is to use, throughout the optimization process, both the true models and the surrogated ones, in a way that reduces the total computational time, without loosing in precision. Due to the necessity to limit the number of training samples it is very difficult to construct an initial approximate model that is globally correct. Likely the approximation will bring the optimization algorithm to false optima, which are optima of the approximate model but not of the true functions. In order to avoid finding false optima, or losing some of the optima, in this work the concept of model management or evolution control (EC) is used.

Jin et $\mathrm{al}^{29}$ in their paper propose two methods: a) individual-based control and b) generation-based control. In the first approach, $n_{v}$ individuals in the current population are chosen and evaluated with the true model. In the latter, the whole population is evaluated with the real model, every $n_{g c y c}$ generations, for $n_{g v}$ generations, where $n_{g v}<n_{g c y c}$. The individuals evaluated with the true model are then introduced into the dataset in order to locally improve the surrogated model in the promising regions.

The method adopted for this work is a mix of both kinds of evolution control. Fig. 2 summarizes the whole optimization process. The MOO optimization algorithm MOPED is integrated with an external procedure that monitors the status of the approximated models. At the end of each iteration (generation), the external procedure checks if an updated version of the approximated model is ready and available. If the approximated model was updated, then all the individuals in the current population are re-evaluated and re-classified with the updated model, before the Parzen distribution is updated and sampled. If the approximated model was not updated, because, for example, a CFD computation is still running, and the difference between the generation of the previous update and the current generation is $n_{\text {gcyc }}$, then MOPED pauses and waits for a new update.

In an asynchronous way, an additional external procedure (bottom right block in Fig. 2) manages the training and updating of the approximated model. This procedure needs as input a list of system models ordered by increasing level of fidelity and a scheduling report detailing how and when the different models should be used. Then it extracts for each optimal trajectory the matrix $\mathbf{S}_{\text {opt }}=\left[l, w, n, \theta, R_{n}, \alpha, \mathbf{v}, \mathbf{H}\right]$. Each row in matrix $\mathbf{S}$ corresponds to point along the trajectory. Each row in $\mathbf{S}$ is then compared to the values in $D B_{\text {train }}$, the matrix of points in the database used to train the ANN. The procedure works as follows:

- At generation 0, it trains a first ANN using the low-fidelity model (fidelity level 0) and then passes the ANN to MOPED (process Initialize App 0 in figure 2); $D B_{\text {train }}$ is initialized;

- At each subsequent generation:

1. Initialize counter $i_{c}=0$;

2. while $i_{c}<=n_{t}$

(a) extracts from the population a sampled trajectory and estract $n_{o}$ operative points;

(b) for $\mathrm{i}=1 \ldots n_{\mathrm{o}}$ 
- compute the minimum Euclidean distance $d_{s l, i}=\min _{j}\left\|S_{o p t, i}-D B_{\text {train }, j}\right\|$ where $j$ loops over all the points in the database (the rows of $D B_{\text {train }}$ );

- if $d_{s l, i}>d_{m i n, s l}$ then the point $S_{o p t, i}$ is evaluated and immediately inserted into the database $D B_{\text {train }}$, and $i_{c}=i_{c}+1$; all the solutions in the database that were computed with a lower fidelity model and have $d_{s l}<d_{m i n, l l}$ are discarded from future updates of the approximating model;

- if $i_{c}=n_{t}$ interrupt loops

- Every $n_{g l}$ generations of the global optimizer, it increases the level of fidelity of the model.

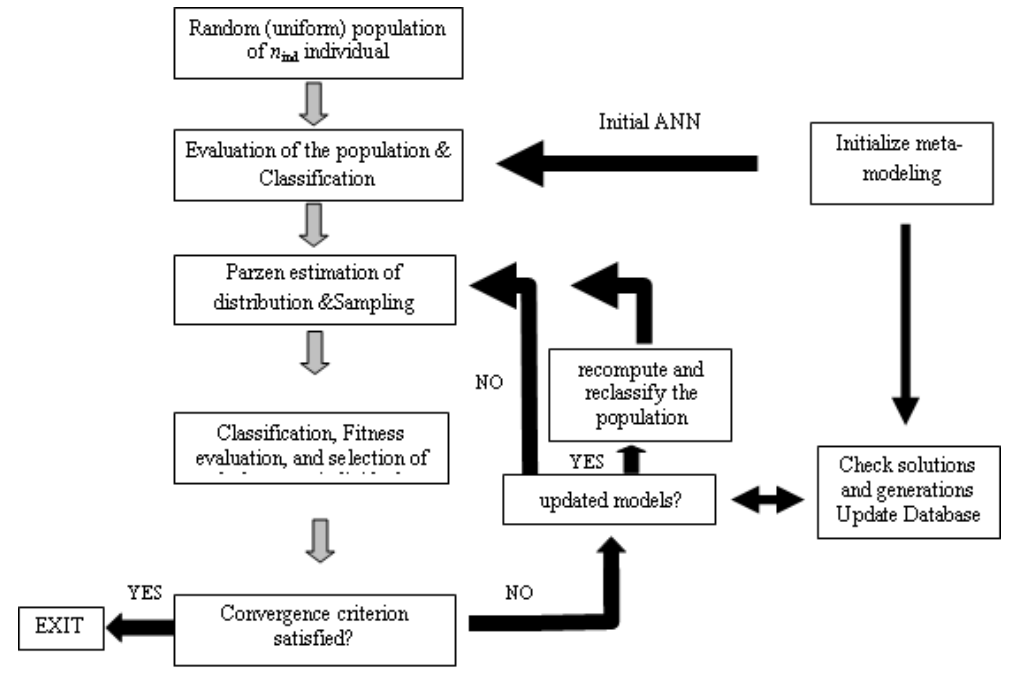

Figure 2: MOPED with evolutionary control modification and independent approximator handler.

\section{III.D. Surrogate Model}

General principia of evolution control do not depend on the particular approximation technique, which is used with, but, of course, the adopted approximation approach strongly affects the application of EC. In this work the surrogate model is an Artificial Neural Network (ANN) approximators. In particular, a generic Multi Layer Perceptron (MLP) ANN with one hidden layer was used. The training process is based on a Bayesian regularization back-propagation, ${ }^{30}$ which limits any overfitting problem. The initial training is performed offline, therefore its speed is of secondary importance. The idea is that the computational cost of the ANN online update is negligible if compared to a call to the high-fidelity model. The inputs to the ANN approximator are the 5 geometric parameters, the angle of attack, the speed, and the altitude. The outputs are the coefficient of lift, $C_{L}$ and drag, $C_{D}$. The networks are trained to reach a mean squared error of $5 \%$ on the normalized training output.

\section{Optimization Results}

The design space for problem (14) is defined by the following bounds on the design parameters: the nominal length $l \in[0.9,1.5][\mathrm{m}]$, the nominal width $w \in[0.4,0.9][\mathrm{m}]$, the exponent $n \in[0.2,0.7]$, the angle $\theta \in[6,11,9][\mathrm{deg}]$, the radius of the nose $R_{n} \in[0.0115 l, 0.026 l]$,the thickness of the TPS at the nose $L_{T P S} \in$ $[0.05,0.15][\mathrm{m}]$. The angle $\beta$, as defined in section II.A, is kept fixed to $12 \mathrm{deg}$.

The trajectories are discretized by 5 elements, with 7 nodes for the states, and 6 Gauss integration points. The bounds on the variables of the trajectory optimization are: total time $T_{t o t} \in[500,6500][s]$, angle of attack $\alpha \in[0,20][\mathrm{deg}]$, radius $r \in\left[6.36710^{6}, 6.4710^{6}\right][\mathrm{m}]$, longitude $L \in[-100,20.9559][\mathrm{deg}]$, latitude $\phi \in$ $[-60,68.0767][\mathrm{deg}]$, speed $v \in\left[10^{2}, 10^{4}\right][\mathrm{m} / \mathrm{s}], \theta_{p} i n[-10,10][\mathrm{deg}]$, heading angle $\xi \in[-225.7396,-55.7396][\mathrm{deg}]$.

The initial conditions (12) are imposed as $x_{0}=\left[R_{E}+10^{5}, 20.9559,68.0767,7700,-0.63247,-145.7641\right]^{T}$, where $R_{E}$ is the mean radius of the Earth, and $r_{f}=25000 \mathrm{~m}$ and $r_{\min }=15000 \mathrm{~m}$. 
The MOO process was carried out for 50 generations with a population of 60 individuals. The initial approximator was built on the basis of 1000 analytic model computations, selected on a randomized Latin Hypercube of the inputs, plus additional 100 super- and subsonic CFD computations to allow the approximator to have an extended range of validity, without needing to extrapolate. The computation of the first database required nearly 700 hours of computational time, distributed on a cluster of 15 linux64 processors (2 days of effective time). The computations of the CFD solver were stopped when convergence was obtained on the aerodynamic forces.

The characteristic parameters of the evolution control were set as follows: $n_{t}=n_{o}=10, n_{g l}=10$, for a total of 2 switches; $n_{g c y c}=5 ; d_{\text {min,sl }}=0.25$ (all the inputs are normalized to $[-1,1]$ ); $d_{\text {min,ll }}=0.5$ for the first 30 generations, and then is increased to 1.0 .

At level 1, which is considered from generation 10, CFD computations were used to verify trajectory points up to $50 \mathrm{~km}$. At level 2 the altitude limit for the use of CFD computation is increased to $90 \mathrm{~km}$, even if the validity of the models at this altitude is at least questionable.

During the computation, until generation 40, the solutions obtained with the CFD model increased up to 450 , allocated in the promising region of the search space, while the analytical ones, used to build the ANN approximator, decreased to nearly 550 .

After 50 generations, the optimization code was able to give an acceptable approximation of the Pareto front as shown in Fig. 3. The sparsity and irregularity of the front approximation were expected, since the objective functions, which are the outcome of the Monte-Carlo sampling, are extremely noisy. Moreover, the speed and accuracy of convergence of the trajectory optimization loop is quite sensitive to shape parameters. Better converge could be obtained with a better initial guess for the control law. In this implementation, however, the first guess is equal for every individual $\left(t_{\max }=1500 \mathrm{~s}\right.$, incidence linearly decreasing from 18 to $16 \mathrm{deg})$.

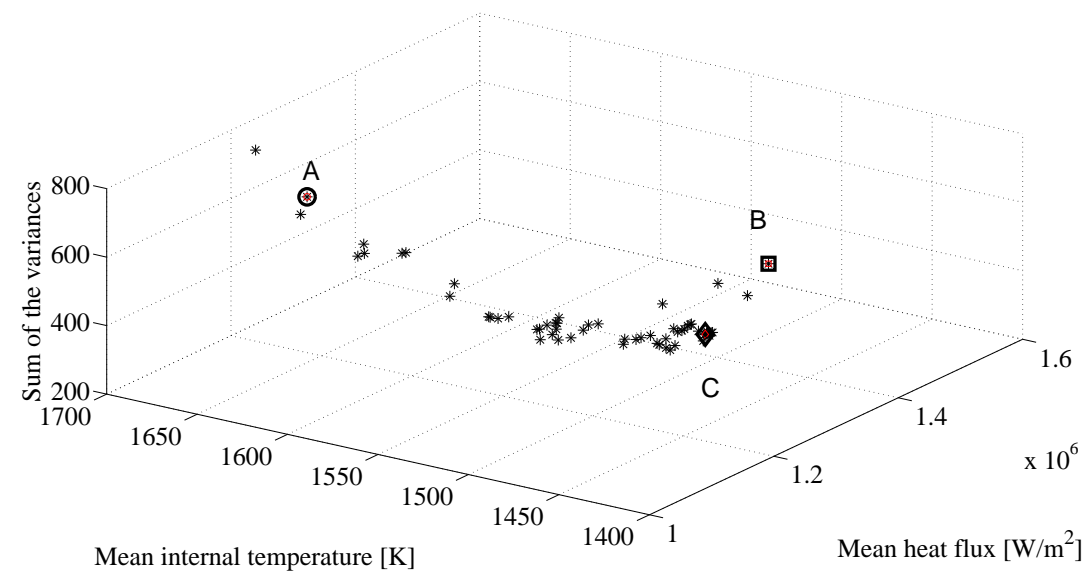

Figure 3: Approximation of the Pareto Front after 50 generations

In Fig, 4 individual $\mathrm{A}$ minimizes the mean value of the maximum heat flux, while individual $\mathrm{B}$ is to be the most robust one, minimizing the sum of the variances. The shape of individual A, which is the most common one, corresponds to a narrow and blunt body. The shape of individual B corresponds to a wider body (see Fig. 4).

The optimization is driven by the easiest way to reduce, or limit, the heat flux at the nose, i.e. increasing the radius of the nose. This is apparently due to the use of the analytical model, which overestimates the performance of the vehicle when the radius of the edges becomes considerably big. The analytical model still retain an impact on the response of the ANN model even if the use of simplified solutions is limited toward the end of the optimization process.

The design parameters for solutions $\mathrm{A}$ and $\mathrm{B}$ are:

- Solution A: $l=1.4918 ; w=0.5762 ; n=0.2052 ; \theta=11.4747 ; R_{n}=0.037447 ; L_{T P S}=0.068935$

- Solution B: $l=1.49915 ; w=0.78706 ; n=0.4348 ; \theta=11.1824 ; R_{n}=0.03755 ; L_{T P S}=0.13719$ 


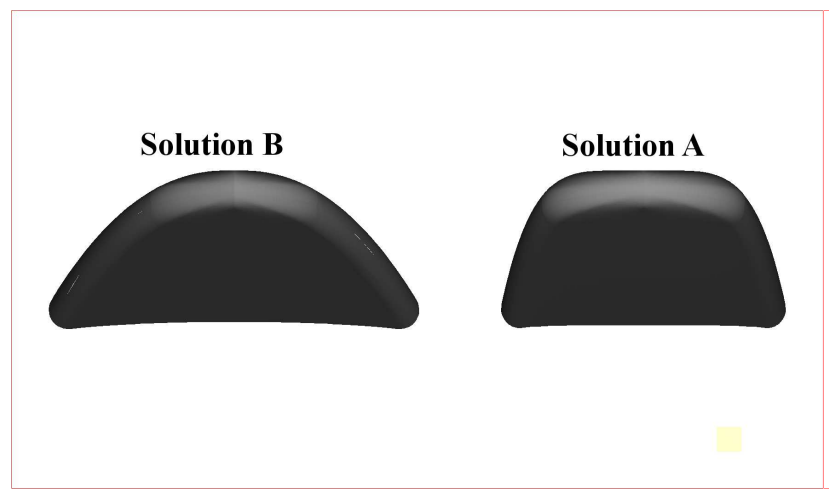

(a) Front view

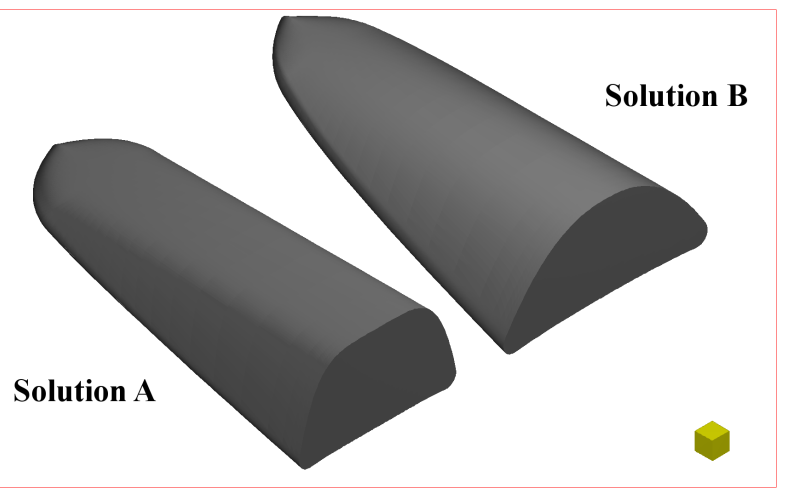

(b) 3d View

Figure 4: Optimal solutions: individual A minimizes the mean value of the maximum heat flux, while individual B minimizes the sum of the variances.

Figure 5 shows the nominal, deterministic trajectories of the two selected individuals. Individual A, about $20 \%$ lighter then solution $\mathrm{B}$, is able to follow a higher re-entry path in the critical part of the trajectory, limiting the heat loads (figure 6), which results to be comparable to what can be found in literature. ${ }^{4,21}$ On the other hand, solution A is evidently more sensitive to changes in the aerodynamic model and in the characteristics of the thermal protection material.

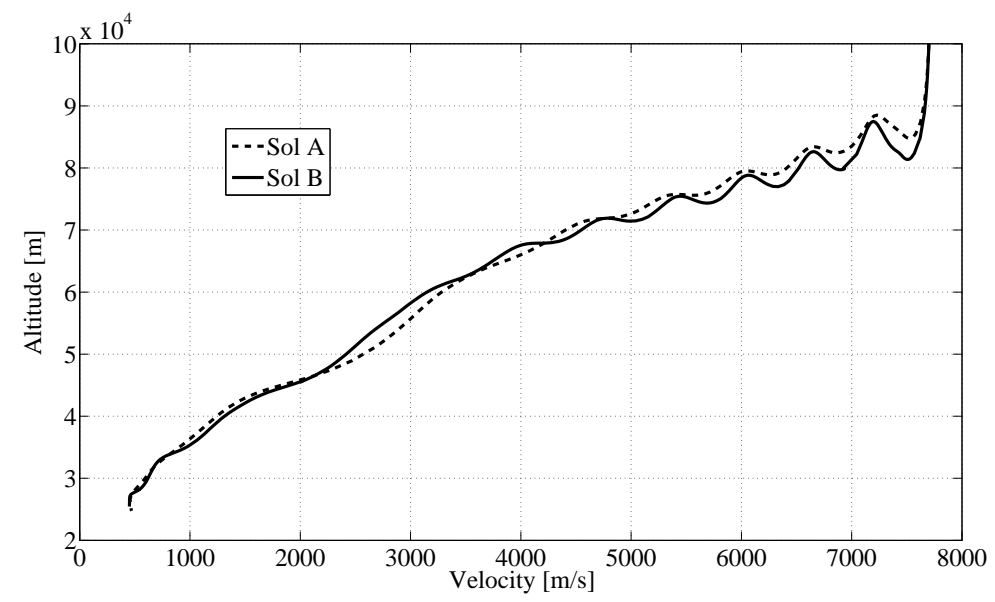

Figure 5: Trajectories of the selected optimal solutions.

The trajectory optimization process, and, as a consequence, the shape of the vehicles are strongly affected by the model of the internal temperature. Vehicle like solution A are slightly lighter than solutions minimizing the temperature (sol C in the figure 3), substantially because of the smaller amount of TPS material at the nose. This allows solutions of type A to follow a higher path, but at the same time having a thinner layer of thermal protection material at the nose means higher internal temperatures and higher temperature variability due to the simulated uncertainties on the characteristics of the material.

The constraint on the final altitude and the bounds on the state variables strongly affect the optimal control law as well. As can be seen in Fig. 7, at the end of the trajectory a sudden pitch-down is required to satisfy the final constraint.

The vehicle passes through the critical part of the atmosphere with an angle of attack which is considerably larger than zero, meaning that the stagnation point is not on the nose but more down-stream on the pressure side. For a real application, it is likely that a blunt-body shape similar to solution A, would require an even higher value of the angle of attack. 


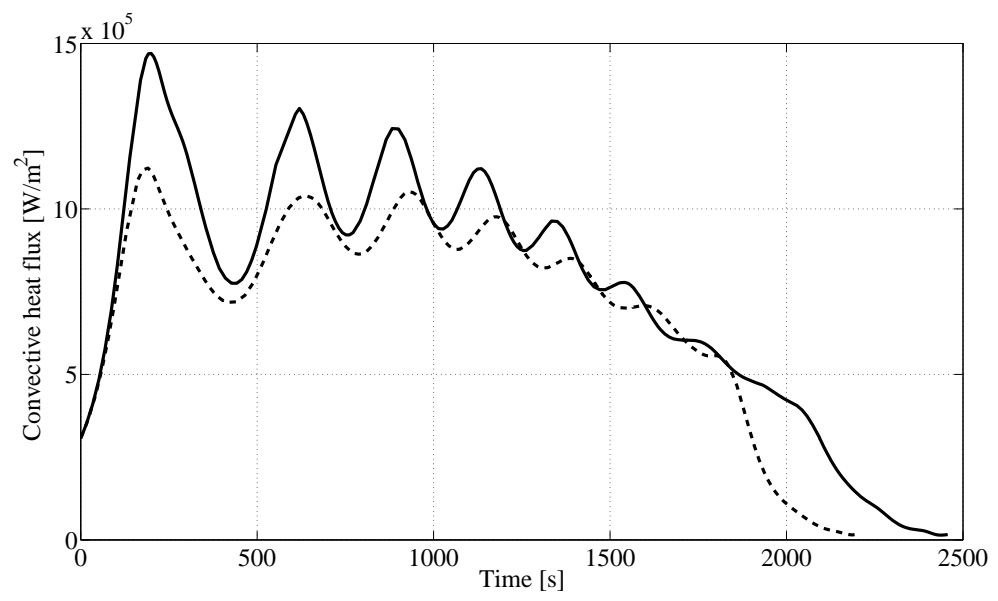

Figure 6: Convective heat flux of the selected optimal solutions.

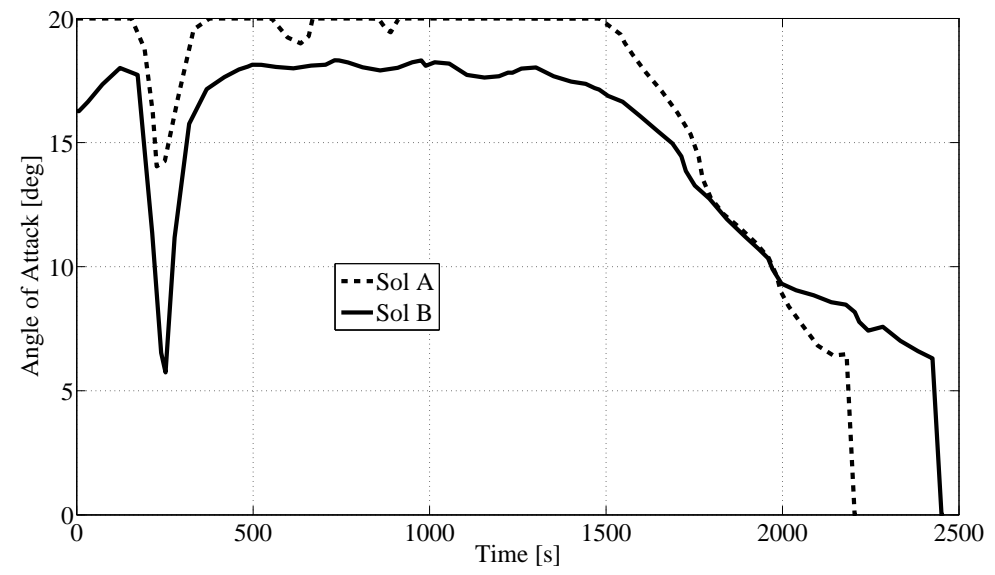

Figure 7: Angle of attack control law.

\section{Conclusions}

This paper addresses the robust multi-disciplinary design of a small scale unmanned space vehicle for re-entry operations. An algorithm was developed, which combines a global, population based solver with a direct transcription method for optimal control problems. Uncertainties on the aerodynamic model and on some characteristics of the thermal protection material are introduced into the vehicle model, and the evolutionary part of the code searches for shapes minimizing the mean value of the heat flux at the nose, the maximum internal temperature and the weighted sum of their variances. The control problem solver is interfaced with an ANN that gives the aerodynamic characteristics of the vehicle on the basis of geometry parameters and operational conditions. The neural network is trained and upgraded by an evolution control procedure, which uses a multi-fidelity approach, in order to limit as much as possible the use of expensive, high fidelity CFD computations.

The whole process was able to detect realistic optimal shapes, with a heating load at the nose that can be considered comparable to what is tolerate by last generation UHTC protection materials.

As expected, such a small vehicle cannot have sharped edges compared to the reduced and compact dimensions, and as a consequence can re-enter with an angle of attack which is considerably larger than zero. This means that the initial hypothesis that the maximum heating should be at the nose is over-conservative, and a correct prediction of the real heating should be obtained by models of higher fidelity, which combines medium- to high-fidelity aero-thermal dynamic computations. 
Moreover, the analytical method resulted misleading, even if its use toward the end of the design process was minimal. All these aspects will be considered in future works, together with a more careful tuning of the approximator and different shape models.

\section{References}

${ }^{1}$ Russo, G. and Matteis, P. D., "PRORA-USV: Two Flight Mission Exploring Transonic Conditions," Proccedings of the 15th AIAA International Space Planes and Hypersonic Systems and Technologies Conference, AIAA, Dayton, Ohio, USA, 2008, pp. AIAA 2008-2660.

${ }^{2}$ Savino, R. and Serpico, D. P. M., "Numerical and Experimental Investigation of PRORA USV Subsonic and Transonic Aerodynamics," Journal of Spacecraft and Rockets, Vol. 43, No. 3, 2006, pp. 575-584.

${ }^{3}$ Russo, G., "USV Program Status 2009," Proceedings of the 16th AIAA/DLR/DGLR International Space Planes and Hypersonic Systems and Technologies Conference, AIAA-2009-7269, Wahington, DC, 2009.

${ }^{4}$ Viviani, A. and Pezzella, G., "Heat Transfer Analysis for a Winged Reentry Flight Test Bed," International Journal of Engineering, Vol. 3, No. 3, 2009, pp. 330-345.

${ }^{5}$ Squire, T. and Marschall, J., "Material property requirements for analysis and design of UHTC components in hypersonic applications," Journal of European Ceramic Society, Vol. 30, No. 1, 2010, pp. 2239-2251.

${ }^{6}$ Strohmeyer, D., Eggers, T., and Haupt, M., "Waverider Aerodynamics and Preliminary Design for Two-Stage-to-Orbit Missions, Part 1," Journal of Spacecraft and Rockets, Vol. 35, No. 4, 1998, pp. 450-458.

${ }^{7}$ Heinze, W. and Bardenhagen, A., "Waverider Aerodynamics and Preliminary Design for Two-Stage-to-Orbit Missions, Part 2," Journal of Spacecraft and Rockets, Vol. 35, No. 4, 1998, pp. 459-466.

${ }^{8}$ Starkey, R. P. and Lewis, M. J., "Analytical Off-Design Lift-to-Drag-Ratio Analysis for Hypersonic Waveriders," Journal of Spacecraft and Rockets, Vol. 37, No. 5, 2000, pp. 684-691.

${ }^{9}$ Umakant, J., Sudhakar, K., Mujumdar, P., and Rao, C. R., "Ranking Based Uncertainty Quantification for a Multifidelity Design Approach," Journal of Aircraft, Vol. 44, No. 2, 2007, pp. 410-419.

${ }^{10}$ Doherty, J. J., Dean, S. R. H., Ellsmore, P., and Eldridge, A., "A Multi-Fidelity Approach for Supporting Early Aircraft Design Decisions," Collaborative Product and Service Life Cycle Management for a Sustainable World, edited by R. Curran, S. Y. Chou, and A. Trappey, Springer London, London, 2008, pp. 267-279.

${ }^{11}$ Choi, S., Alonso, J., Kroo, I., and Wintzer, M., "Multifidelity Design Optimization of Low-Boom Supersonic Jets," Journal of Aircraft, Vol. 45, No. 1, 2008, pp. 106-118.

${ }^{12}$ Choi, S., Alonso, J., and Kroo, I., "Two-Level Multifidelity Design Optimization Studies for Supersonic Jets," Journal of Aircraft, Vol. 46, No. 3, 2009, pp. 776-790.

${ }^{13}$ Robinson, T., Eldred, M., Willcox, K., and Haimes, R., "Surrogate-Based Optimization Using Multifidelity Models with Variable Parameterization and Corrected Space Mapping," AIAA Journal, Vol. 46, No. 11, 2008, pp. 2814-2822.

${ }^{14}$ Singh, G. and Grandhi, R., "Mixed-Variable Optimization Strategy Employing Multifidelity Simulation and Surrogate Models," AIAA Journal, Vol. 48, No. 1, 2009, pp. 215-223.

${ }^{15}$ Vasile, M. and Bernelli-Zazzera, F., "Optimizing Low-Thrust and Gravity Assist Maneuvres to Design Interplanetary Trajectories," The Journal of the Astronautical Sciences, Vol. 51, No. 1, 2003, January-March 2003.

${ }^{16}$ Vasile, M. and Bernelli-Zazzera, F., "Targeting a Heliocentric Orbit Combining Low-Thrust Propulsion and Gravity Assist Manoeuvres," Operational Research in Space 85 Air, Vol. 79, Kluwer Academy Press, ISBN 1-4020-1218-7, 1st ed., 2003.

${ }^{17}$ Fornberg, B., A Practical Guide to Pseudospectral Methods, Cambridge University Press, 1998.

${ }^{18}$ Chapman, C., High speed flow, Cambridge University Press, 2000.

${ }^{19}$ White, F., Viscous Fluid Flow, McGraw-Hill, New York, 1974.

${ }^{20}$ Aupoix, B. and Cousteix, J., "Real Gas Effects in Two- and Three-Dimensional Hypersonic, Laminar Boundary Layers," Kluwer Academic Publishers, 2008, pp. 293-340.

${ }^{21}$ Savino, R., De Stefano Fumo, M., Paterna, D., and Serpico, M., "Aerothermodynamic study of UHTC-based thermal protection systems," Aerospace Science and Technology, Vol. 9, 2005, pp. 151-160.

${ }^{22}$ Anderson, J., Hypersonic and High-Temperature Gas Dynamics (Second Edition), AIAA, 2006.

${ }^{23}$ K.Cowart and J.Olds, "Integrating Aeroheating and TPS Into Conceptual RLV Design," AIAA, AIAA 99-4806, 1999.

${ }^{24}$ Hankey, W. L., Re-entry Aerodynamics (AIAA Education Series), AIAA, 1988.

${ }^{25}$ Costa, M. and Minisci, E., "MOPED: a Multi-Objective Parzen-based Estimation of Distribution algorithm," Evolutionary Multi-Criterion Optimization. Second International Conference, EMO 2003, edited by C. Fonseca, P. Fleming, E. Zitzler, K. Deb, and L. Thiele, Vol. 2632 of LNCS, Springer, Faro, Portugal, 08-11 April 2003, pp. 282-294.

${ }^{26}$ Avanzini, G., Biamonti, D., and Minisci, E., "Minimum-Fuel/Minimum-Time Maneuvers of Formation Flying Satellites," Astrodynamics Specialist Conference, AAS 03-654, Big Sky, Montana, 03-07 August 2003.

${ }^{27}$ Deb, K., Pratap, A., Agarwal, S., and Meyarivan, T., "A Fast and Elitist Multiobjective Genetic Algorithm: NSGA-II," IEEE Transaction on Evolutionary Computation, Vol. 6, No. 2, april 2002, pp. 182-197.

${ }^{28}$ Fukunaga, K., Introduction to statistical pattern recognition, Academic Press, 1972.

${ }^{29}$ Jin, Y., Olhofer, M., and Sendhoff, B., "Framework for Evolutionary Optimization with Approximate Fitness Functions," IEEE Transactions on Evolutionary Computation, Vol. 6, No. 5, 2002, pp. 481-494.

${ }^{30}$ MacKay, D., "Bayesian interpolation," Neural Computation, Vol. 4, No. 3, 1992, pp. 415-447. 